\title{
Flexion contractures of the lower limbs with urogenital anomalies
}

Fares Chedid, Mansour Qurashi, Jean Mah

A female infant was born at term to a mother with uncontrolled insulin-dependent diabetes mellitus (IDDM). Blood sugar during the first few weeks of pregnancy was around $300 \mathrm{mg} / \mathrm{dl}$ (15 $\mathrm{mmol} / \mathrm{l})$. The baby was born with flexion contractures of the lower limbs, paralysis and muscle wasting (figure 1). She had normal heart sounds without heart murmur. The anus was imperforated and the urethral end was not visible. The urine and stools were passed through a common cloaca. Renal ultrasound and scanning with technetium-labelled 2,3-dimercaptosuccinic acid (DMSA) revealed left-sided renal agenesis. A voiding cysto-urethrogram showed grade IV vesico-ureteral reflux. A skeletal survey is shown in figure 2. A photograph of the patient taken prone is presented in figure 3.

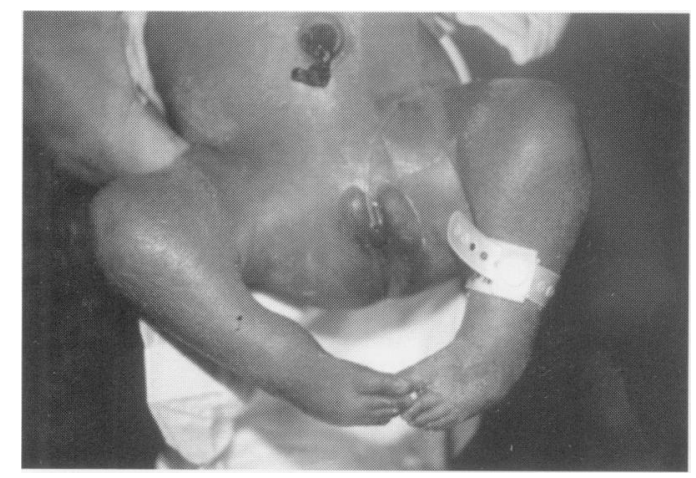

Figure 1

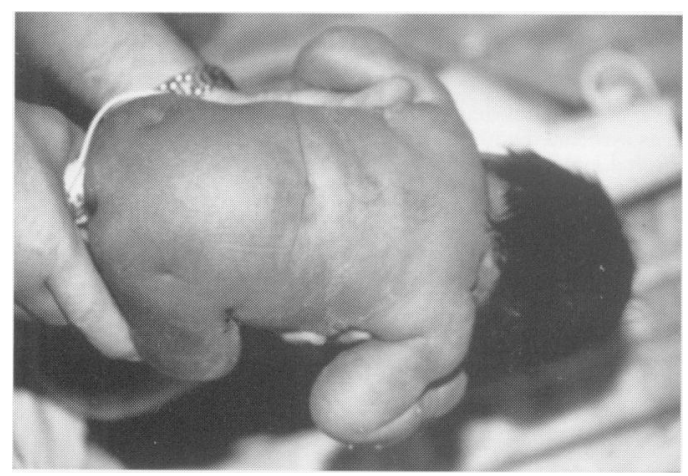

Figure 3

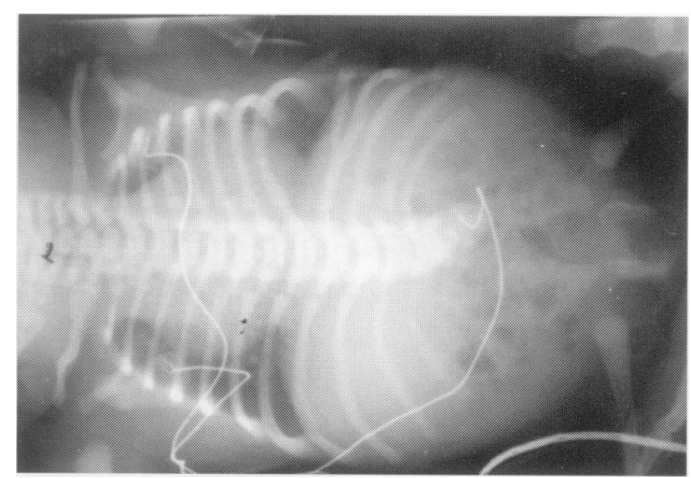

Figure 2

\section{Questions}

1 What is your diagnosis?

2 What is the underlying mechanism?
National Guard King Khalid Hospital, PO Box 9516, Jeddah 21423, Saudi Arabia F Chedid

M Qurashi

J Mah

Accepted 13 May 1998 


\section{Answers}

QUESTION 1

This patient has a syndrome of caudal regression and cardiomegaly.

\section{QUESTION 2}

The syndrome of caudal regression in this newborn is due to uncontrolled periconceptual IDDM affecting organogenesis. The cardiomegaly is similarly due to hyperglycaemia during pregnancy.

\section{Discussion}

The patient has an extreme hypoplasia of the lower body pole with aplasia of the spine below L1 (figure 2), paraplegia and contractures. The syndrome is named caudal regression or caudal axial mesodermal dysplasia. The insult to the mesoderm occurs before 8 weeks of gestation and maternal diabetes is the major risk factor, being associated with $16 \%$ of cases. ${ }^{12}$ All organs originating from the mesoderm and the lower body pole can be affected to a variable degree. Major associated anomalies include anogenital malformations, unilateral renal agenesis, neurogenic bladder and vesicourethral reflux. ${ }^{3}$ Gluteal skin dimples (figure 3) are frequently associated with the syndrome. They are due to atrophy of subcutaneous and adipose tissues resulting from close contact between the skin and the bone.

Management of caudal regression involves a multidisciplinary team. The goals are to prevent renal complications, to restore the anal canal and urogenital tract, and to rehabilitate the patient.

The emphasis must be put on prevention. Women with diabetes should monitor their glucose levels and defer their pregnancies until

1 Houffin V, Subtil D, Cosson M, et al. Prenatal diagnosis of three caudal regression syndromes associated with maternal diabetes. $f$ Gynecol Obstet Biol Reprod 1996;25:389-95.

2 Stuart JM, Stall S. Familial caudal regression anomaly and maternal diabetes. $\mathcal{F}$ Med Genet 1979;16:17-20.
Problems associated with diabetes in pregnancy

Hyperglycaemia during conception and organogenesis

- caudal regression

- neural tube defects

- abdominal wall defects

- ventricular septal defect

- trisomy 21

- other malformations

Gestational diabetes (resistance to insulin after 24 weeks gestation)

- macrosomia and birth trauma

- cardiac hypertrophy, especially septal hypertrophy

- metabolic disturbances, eg, hypoglycaemia, hypocalcaemia

- polycythaemia

- hyaline membrane disease

their glycosylated haemoglobin is relatively normal. This method is very effective in reducing the occurrence of caudal regression, as well as other diabetes-associated malformations such as spina bifida and omphalocoele. Normoglycaemia should be maintained during pregnancy in order to prevent myocardial hypertrophy, macrosomia, dystocia and other associated complications.

\section{Final diagnosis}

Congenital caudal regression and cardiomegaly due to uncontrolled periconceptual diabetes.

Keywords: caudal regression; mesodermal dysplasia; diabetes mellitus; cardiomegaly

3 Boemers TM, Van Gool JD, de Jong TP, Bax KM. Urodynamic evaluation of children with caudal regression syndrome (caudal regression sequence). $\mathcal{F}$ Urol 1994;151: 1038-40. 